\title{
Should photographic triage become common practice?
}

\author{
Nicole Thomas ${ }^{1}$
}

\section{A commentary on}

\section{Davies A, Howells R, Lee S M G, Sweet C J, Dominguez-}

\section{Gonzalez S.}

Implementation of photographic triage in a paediatric dental, orthodontic and maxillofacial department during COVID-19. Int J

Paediatr Dent 2020; DOI: 10.1111/ipd.12773.

\begin{abstract}
Design Cross-sectional, exploratory study collecting photographic referral data over three months.

Sample selection Paediatric referrals to the orthodontic and maxillofacial department at Alder Hey Hospital during the COVID-19 pandemic.

Data analysis Photographic referrals were analysed using Microsoft Excel to determine treatment outcomes, including 'not to see', 'plan to see' and 'need to see'. Demographic information collected and analysed were patient age, gender, referral source and presenting complaint.

Results In total, 220 photographic referrals were received, with swelling (30\%) and dental trauma (27\%) being the most common presenting complaints. Fifty-seven percent of the referrals were not seen, $23 \%$ were seen semi-urgently and $20 \%$ booked for outpatient review. Of those seen, seven children were examined elsewhere, with 44 receiving face-to-face consultations at Alder Hey Children's Hospital, with eight being admitted.

Conclusions Photographic triage has potential to be a useful adjunctive assessment tool for new patient referrals, with secondary benefits for training junior staff and for populations who already find access to dental services challenging. Feasibility studies on the application should be seriously considered. However, the unique circumstances which resulted in the development of a photographic triage method need to be considered when generalising its use to normal working conditions.
\end{abstract}

\section{Commentary}

In March 2020, general dental practitioners were advised to cease all face-to-face contact due to the COVID-19 pandemic. ${ }^{1}$ Following this abrupt disruption to primary care dental services, patients with acute dental, orthodontic or maxillofacial problems had limited options. For primary care, patients could attend one of the newly commissioned urgent dental centres and/or an oral maxillofacial department for secondary or tertiary care. This paper

\section{GRADE rating}

\section{Practice points}

- Assessing children remotely may help break down barriers, particularly for children with sensory challenges who may otherwise avoid accessing dental services.

- Photographic triage, including monitoring and reviewing preexisting conditions, could reduce the opportunity costs for both clinicians and families.

- Reducing the need for face-to-face encounters significantly reduces the risk of spreading disease, particularly during the COVID-19 pandemic.

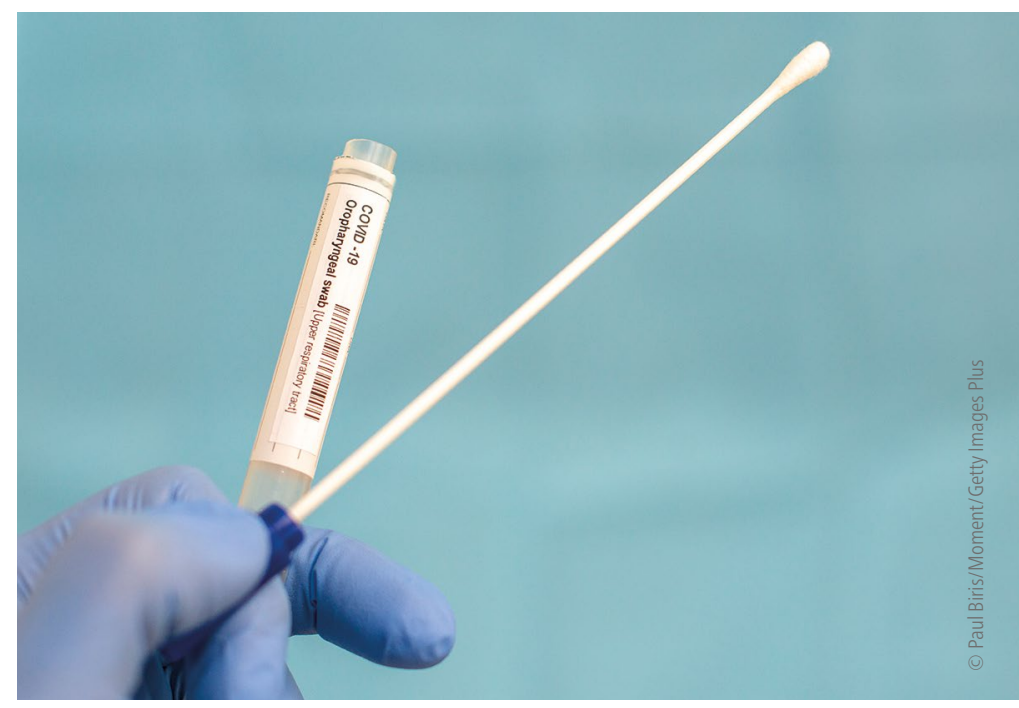

describes descriptive findings on the usefulness of a photographic triage method to determine treatment needs in children referred by parents and a wide range of health professionals within the hospital and in the wider community.

Alder Hey Children's Hospital serves a population of approximately one million children aged 16 and under, and has a combined dental, orthodontic and oral maxillofacial surgery department. After ceasing all routine outpatient appointments and elective general anaesthetic procedures, a combined telephone and photographic triage system was rapidly developed so that referrals for acute dental conditions could be triaged. Data were collected between March and June 2020. These included patient age and gender, referral source, date of referral, presenting complaint and triage choice. The three triage options were 'not to see', 'plan to see' and 'need to see'.

A total of 220 photographic referrals were received in relation to 190 children (107 male, 83 female), aged between 3 months to 21 years old ( $\mu=7$ years). Swelling and dental trauma were the most common presenting complaints (57\%). For 23 children, images were received on more than one occasion. Screening was carried out by the oral and maxillofacial surgery dental core trainees, paediatric dental registrars and nursing staff. Following 
a full clinical history, images were requested if necessary, with a consultant making the final decision on the treatment outcome. Over half the referrals were not seen. A total of 44 children received a face-to-face consultation, with eight being admitted to the hospital.

Despite its considerable limitations, this paper is important as it highlights both useful applications of a photographic triage method where access to services may be severely limited as well as how a photographic triage system could improve referral systems generally. Secondary benefits to staff training were also found. The study also highlights the distinct paucity of research in this area.

Further research is warranted to enhance systems that may now be outdated. ${ }^{2}$ However, care should be taken when comparing the highly unusual circumstances which led to the development of this photographic triage method to general working conditions. For example, parent carers would not ordinarily be involved in the direct referral of their children to services. There was also a noticeable increase in dental trauma noted by the hospital, leading to lockdown and children playing in different environments to school being hypothesised as the cause.

\section{Author affiliation}

${ }^{1}$ Faculty of Health, University of Plymouth, Plymouth, UK. Correspondence to: Nicole Thomas

\section{References}

1. Chief Dental Officer. Preparedness for Primary Dental Care. 2020. Available at www.england.nhs.uk/coronavirus/wp-content/uploads/sites/52/2020/03/ issue3preparednessletterforprimarydentalcare25march2020.pdf (accessed July 2020)

2. Hogan R, Goodwin M, Boothman N, lafolla T, Pretty I A. Further opportunities for digital imaging in dental epidemiology. I Dent 2018; 74 Suppl 1: S2-S9.

Evidence-Based Dentistry (2021) 22, 52-53.

https://doi.org/ 10.1038/s41432-021-0170-6 